\title{
ESTUDO COMPARATIVO ENTRE BUPIVACAÍNA 0,5\% E ROPIVACAÍNA 0,5\% NO BLOQUEIO DOS NERVOS PALMARES DE EQUINOS HÍGIDOS
}

\section{COMPARATIVE STUDY BETWEEN BUPIVACAINE 0,5\% AND ROPIVACAINE 0,5\% IN PALMAR NERVES BLOCK OF HEALTHY HORSES}

\author{
Pierre Barnabé Escodro, Cicero Ferreira Oliveira, Josealdo Tonholo, Ticiano Gomes \\ Nascimento, Ricardo G.O. Vilani, Fábio R.P. Bruhn \\ *Correspondência: pierre.vet@gmail.com:
}

RESUMO: O bloqueio dos nervos palmares é uma técnica de anestesia regional que possibilita realização de diagnóstico de claudicações e analgesia na região distal do membro torácico de equinos. Este trabalho objetivou comparar a eficácia da bupivacaína 0,5\% (BUPI) e ropivacaína 0,5\% (ROPI) sem vasoconstritor no bloqueio dos nervos palmares, guiado por eletroestimulação. Após colocação de ferradura indutora de claudicação no membro torácico direito, proporcionando claudicação grau (GC) 3, e localização do nervo palmar: cinco equinos adultos hígidos e não portadores de processos inflamatórios, foram submetidos à infiltração de $5 \mathrm{~mL}$ de BUPI e ROPI em cada nervo, avaliando-se o GC a cada 15 minutos, até o tempo de retorno ao GC 3. Todos os animais participaram dos dois grupos, com intervalo entre bloqueios de 45 dias. Foram comparados os seguintes parâmetros de resposta anestésica: tempo de latência sensitiva ( $T L)$, tempo de latência para efeito máximo (TLM), tempo do efeito sensitivo (TE) e tempo de duração do efeito máximo (TEM) por meio do teste de Wilcoxon. A técnica de eletroestimulação nervosa foi eficaz em $100 \%$ dos animais. O TL médio da Bupivacaína foi de 5,0 minutos, aproximadamente dez minutos menor que o da ROPI $(p<0,05)$, mostrando que 0 primeiro fármaco apresenta ação mais rápida para bloqueios perineurais diagnósticos ou analgésicos pré-cirúrgicos. Em relação ao TLM e TEM não foi observada diferença entre os anestésicos $(p>0,05)$, entretanto, em relação ao $T$, a bupivacaína apresentou média de 230 minutos, maior tempo de analgesia em relação à $\operatorname{ROPI}(p<0,05)$. A partir desta pesquisa, conclui-se que o uso de bupivacaína apresentou efeitos analgésicos superiores à ropivacaína nos bloqueios dos nervos palmares de equinos hígidos.

Palavras-chave: claudicação; membros torácicos; anestesia perineural; anestésicos locais

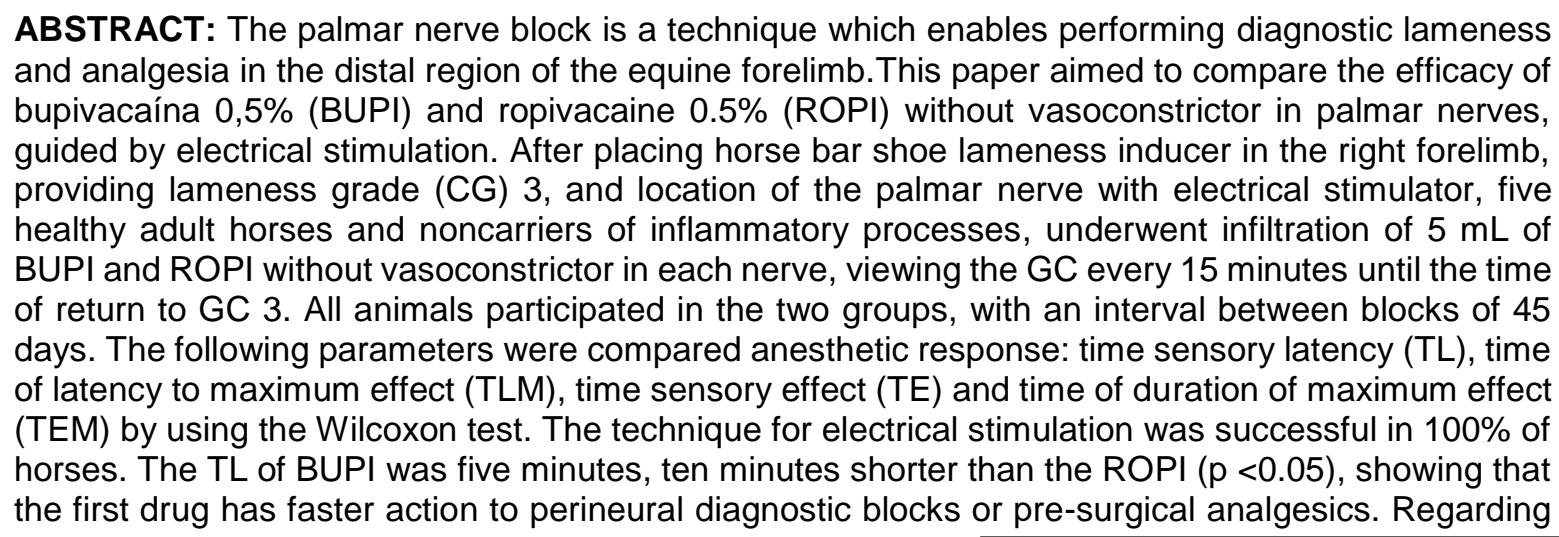

Recebido em 24/11/2014 Aprovado em 21/082015 
the TLM and TEM no difference between the anesthetics ( $p>0.05)$, however, compared to the TE, BUPI was time of 230 minutes, also showed longer duration of analgesia than ROPI $(p<0.05)$. From this research, it is concluded that the use of bupivacaine showed superior analgesic effects of ropivacaine to palmar nerves block of the healthy horses.

Key Words: lameness, forelimb, perineural anaesthesia, local anesthetic 


\section{INTRODUÇÃO}

A utilização da analgesia perineural diagnóstica para claudicações de equinos é bastante aceita na prática veterinária, sendo satisfatória nos casos em que, mesmo após anamnese eficiente e acurado exame clínico, a origem e a região específica desta claudicação ainda não tenham sido identificadas (Gilbson e Stashak, 1989; Lopes, 2011). Também tem crescido o uso da analgesia perineural trans e póscirúrgica, respectivamente diminuindo doses de fármacos nas anestesias gerais e fornecendo alívio em afecções dolorosas com sede distal do membro torácico, como em casos de laminite e fratura de falange distal (Nicoletti et al., 2007; Escodro et al., 2011), inclusive com desenvolvimento de técnica de bloqueio anestésico contínuo do nervo palmar (Driessen et al., 2008).

Dessa forma, houve um incremento na introdução de fármacos anestésicos de longa duração na anestesia perineural de equinos, como é - caso da bupivacaína e ropivacaína (Driessen et al., 2008). Porém ainda são insuficientes as pesquisas que tragam estudos comparativos sobre seus efeitos em nervos específicos, visando suprir ou aliviar quadros álgicos em pósoperatórios; ou em afecções clínicas do membro com evolução dolorosa prolongada (Zarucco et al., 2010).

$O$ anestésico local de longa ação mais utilizado na prática equina é a bupivacaína, com tempo de latência e duração de efeito de 10 a 30 minutos e 180 a 480 minutos respectivamente, além de lipossolubilidade de 3420 (Lamount, 2008), sendo que Adami et al. (2011) obtiveram média superior a quatro horas em nervos ciáticos de cabras. Já a ropivacaína, quimicamente homóloga à mepivacaína e à bupivacaína, com lipossolubilidade de 775, apresenta tempos de latência inferior e de efeito semelhante à bupivacaína, porém é mais onerosa (Moyer et al., 2007; Lamount, 2008). Todavia, seu uso é defendido devido às seguintes vantagens: apresentar menor toxicidade nervosa, demonstrar menor potencial arritmogênico em estudos pré-clínicos (Kruijt Spanjer et al., 2011) e obter maior tempo de ação (até treze horas), possivelmente por possuir ação vasoconstritora, clinicamente comprovada em estudos médicos (Lofstrom, 1992; Feldman, 1994). No entanto, Wakoff et al. (2013) concluíram que a bupivacaína a $0,25 \%$ sem vasoconstrictor utilizada em bloqueio de plexo braquial de cães conferiu maior tempo de analgesia e bloqueio motor em relação à ropivacaína, com tempo médio de bloqueio sensitivo de 768,5 \pm 184 minutos, versus $296,9 \pm 82,50$ minutos da ropivacaína.

O objetivo desta pesquisa foi comparar o tempo de latência e a duração do efeito analgésico da bupivacaína $0,5 \%$ ou da ropivacaína $0,5 \%$ no bloqueio dos nervos palmares de equinos hígidos.

\section{MATERIAL E MÉTODOS}

Utilizaram-se cinco equinos adultos hígidos, sem raça definida, sendo quatro fêmeas e um macho com peso médio de $302 \pm 52,21 \mathrm{Kg}$ e idade média de $4,1 \pm 0,89$ anos. Todos os animais foram avaliados clinicamente em relação à ausência de claudicação; e sanidade através de hemogramas completos dentro dos valores indicados para espécie, exames negativos para Mormo e Anemia Infecciosa Equina. O delineamento estatístico experimental foi o "cross-over", caracterizado pela utilização de dois tratamentos (bloqueios anestésicos) aplicados às mesmas unidades experimentais após intervalo de 45 dias. O primeiro momento foi caracterizado pelo bloqueio anestésico palmar com cloridrato de bupivacaína 0,5\% (BUPI); no segundo momento o 
fármaco utilizado foi cloridrato de ropivacaína $0,5 \%$ (ROPI).

Os equinos receberam ferraduras em ambos os membros torácicos, 72 horas antes do experimento, sendo que - esquerdo recebeu ferraduras de modelo padrão, enquanto que no membro anterior direito foi utilizado modelo de ferradura para indução de claudicação, adaptado daquele utilizado por Nicoletti et al. (2007). A ferradura apresentava duas barras e dois orifícios com roscas para parafusos de $6 \mathrm{~mm}$ de diâmetro, denominados como ponto ranilha (PR) e ponto central (PC), conforme ilustrado na Figura 1. A claudicação foi induzida com a colocação do parafuso até oferecer resistência à sola do casco e promover claudicação de grau 3, segundo Stashak (2002), desta forma as ferraduras e parafusos foram moldados individualmente por animal e por ponto de pressão (PC e PR). Os parafusos foram colocados somente no momento da avaliação da claudicação e as ferraduras foram retiradas durante 0 período de intervalo entre os dois tratamentos (BUPI-ROPI) e após o último tratamento (ROPI).

Figura 1- Vista ventral da ferradura utilizada no experimento, com discriminação dos orifícios submetidos a pressão com parafusos de $\phi 6 \mathrm{~mm}$. Modelo de ferradura adaptado de Nicoletti (2007): PC (ponto central) e PR (ponto ranilha). Fonte: Autores.

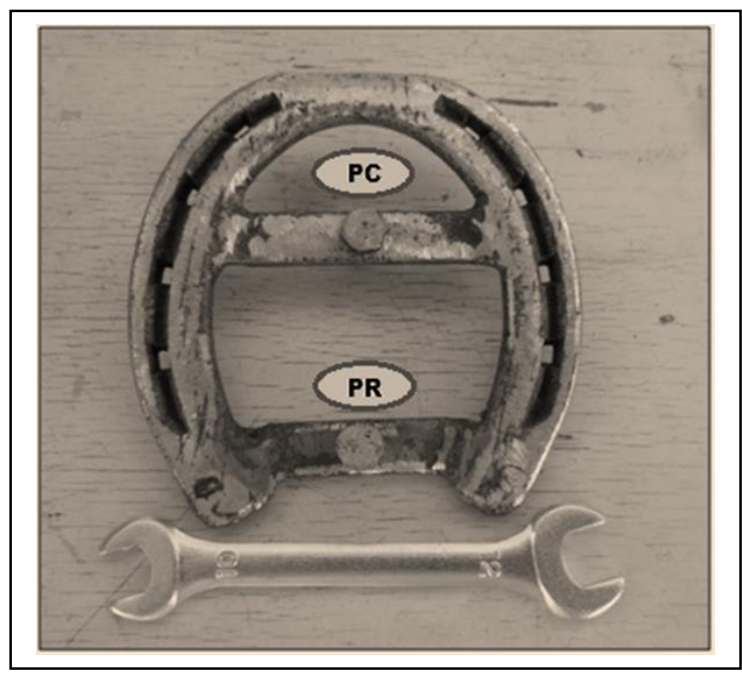

Evidenciada a claudicação grau 3 por dois avaliadores com experiência em claudicação, o membro foi flexionado e a identificação do nervo palmar (NP) foi realizada por meio da palpação bilateral entre o ligamento suspensório do boleto e tendão flexor digital profundo (TFDP) (Nicoletti et al., 2007). A região metacarpofalângica do membro torácico direito foi submetida à antissepsia com etanol a $70 \%$, sem necessidade de tricotomia, procedendo-se a inserção de agulha hipodérmica de calibre $25 \mathrm{G}$ de 10 $\mathrm{cm}$ de comprimento conectada a um eletroestimulador de nervo por via subcutânea (Neurolocalizador MyoTest VI - Amrra Electromedicina - Buenos Aires, Argentina), visando maior precisão para infiltração. Foi utilizada uma corrente inicial de intensidade de $5 \mathrm{~mA}$ e reduzida progressivamente a $0,5 \mathrm{~mA}$, observando a resposta motora dos respectivos grupos musculares na região, buscando obter proximidade média de $0,3 \mathrm{~cm}$ do nervo palmar, não realizando infiltração intrafascicular (risco de axonotmese ou neurotmese), conforme já descrito por Escodro (2011). $O$ volume de infiltração anestésica foi de $5 \mathrm{~mL}$ no nervo palmar medial e $5 \mathrm{~mL}$ no nervo palmar lateral, após obter intensidade de corrente próxima a $0,5 \mathrm{~mA}$ com resposta motora, independendo do tratamento. 
Para o tratamento BUPI foi utilizada a infiltração perineural palmar de cloridrato de bupivacaína a 0,5\% (Neocaína® - Cristalia Produtos Químicos Farmacêuticos Ltda - Itapira, Brasil). Já para o tratamento ROPI houve necessidade de manipulação do anestésico, visto que a apresentação comercial da ropivacaína é na forma de frasco ampola a 1\% (Ropi $®$ - Cristalia Produtos Químicos Farmacêuticos Ltda Itapira, Brasil), procedendo-se 0 acréscimo $10 \mathrm{~mL}$ de água deionizada, perfazendo $20 \mathrm{~mL}$ de solução a $0,5 \%$, realizado em capela de fluxo laminar (Filtracom - Valinhos, SP, Brasil). As soluções foram preparadas no período imediatamente anterior ao procedimento, ficando dessa forma o menor tempo possível armazenada na seringa.

A avaliação da claudicação após a infiltração dos anestésicos foi realizada a cada 15 minutos, sendo os animais trotados sempre pelo mesmo condutor, até o tempo em que o animal retornava ao Grau 3 de claudicação inicial, considerado o tempo de efeito sensitivo (TE). Entre as avaliações, os animais foram mantidos em repouso em grama com cabresto e guia. As avaliações sempre tiveram início no final da tarde, terminando a noite. Manteve-se o critério de avaliação do grau de claudicação baseado na classificação de Stashak (2002), convencionando-se a ausência de claudicação como grau 0 .

Foram calculadas as medianas dos seguintes parâmetros de avaliação da resposta anestésica: 1) Tempo de latência sensitiva (TL): intervalo de tempo, em minutos, entre a infiltração do fármaco e a diminuição do grau de claudicação (Grau 2 ou 1); 2) Tempo de latência para efeito máximo (TLM): intervalo de tempo, em minutos, entre a infiltração do fármaco até a ausência de claudicação (Grau 0); 3) Tempo do efeito sensitivo (TE): Intervalo de tempo, em minutos, que os animais retornam ao Grau 3; 4) Tempo de duração do efeito máximo (TEM): intervalo de tempo em que os animais permaneceram sem claudicar.

Para avaliar possíveis diferenças nos parâmetros TL, TLM, TE e TEM foi utilizado o teste não paramétrico para amostras pareadas de Wilcoxon, depois de verificada ausência de distribuição normal pelo teste de Shapiro-Wilk. Assim, os dados foram descritos por meio de mediana e distância interquartílica (DI), sendo a sua distribuição avaliada por meio de gráficos de Box-plot para cada parâmetro de avaliação de resposta anestésica avaliado. As análises estatísticas foram feitas por meio do software SPSS 20.0. Considerou-se o nível de confiança mínimo de $95 \%$.

\section{RESULTADOS E DISCUSSÃO}

O modelo de ferradura utilizado para induzir claudicação, os comprimentos e diâmetro dos parafusos e a pressão aplicada aos mesmos nos pontos PR e PC, produziu imediata claudicação de apoio em todos os animais desta pesquisa, independentemente do ponto em que foi testado, podendo ser considerado como bom modelo para indução de claudicação por pressão solar. Após a avaliação da claudicação e removida a pressão aplicada na sola pelos parafusos, os animais imediatamente voltaram à locomoção normal, classificados com grau 0 de claudicação. Os testes aplicados não causaram nenhum dano permanente aos animais. O uso do neurolocalizador nos cavalos do mesmo tratamento constatou tempo de respostas analgésicas semelhantes entre os animais, demonstrando a eficácia do equipamento na localização nervosa, como já citado por Futema et al. (1999) em plexos braquiais de cães e por Escodro (2011) em nervos palmares de equinos. Dessa forma, 
neurolocalizador pode ser considerado como ótima ferramenta no intuito de melhorar a precisão em bloqueios palmares de equinos, diminuindo erros inerentes à localização do nervo por meio de palpação e identificação a partir de referências anatômicas regionais.

Os dados obtidos em relação ao TL, TLM, TE e TEM dos tratamentos BUPI e ROPI estão apresentados na Tabela 1, Figuras 2 e 3 . Foram observadas diferenças estatisticamente significativas $(p<0,05) \quad$ entre os anestésicos no TL e no TE, sendo que a bupivacaína apresentou menor TL (mediana $=5(\mathrm{Dl}=0)$ versus mediana $=15$ $(\mathrm{Dl}=0)$ minutos) e maior $\mathrm{TE}$ (mediana $=230 \quad(\mathrm{DI}=0) \quad$ versus mediana=225 ( $\mathrm{DI}=30$ ) minutos) que a ropivacaína. Assim, observa-se que o TL do BUPI foi aproximadamente dez minutos menor que o do ROPI $(p<0,05)$, mostrando que o primeiro fármaco promove analgesia palmar mais rápida para os bloqueios perineurais diagnósticos ou analgésicos. Em relação ao TLM e TEM não foi observada diferença entre os anestésicos ( $p>0,05)$. Outro resultado relevante foi que os animais comportaram-se de maneira idêntica em relação aos graus de claudicação em PC e PR por momento, o que viabilizou demonstração dos resultados unificados, não os diferenciando por ponto de pressão. A partir do experimento, sugere-se que futuras pesquisas possam ser realizadas apenas com um ponto de pressão por ferradura, diminuindo a exposição dos equinos à dor.

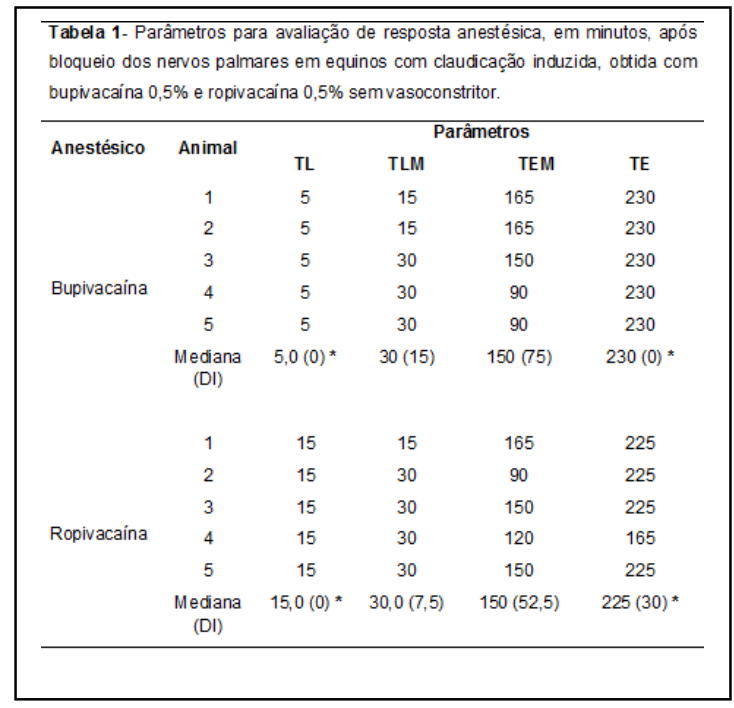

Parâmetros: TL (tempo de latência sensitiva): intervalo de tempo, em minutos, entre a infiltração do fármaco e a diminuição do grau de claudicação (grau 2 ou 1); TLM (tempo de latência para efeito máximo): intervalo de tempo, em minutos, entre a infiltração do fármaco até a ausência de claudicação (grau 0); TEM (tempo de duração do efeito máximo): intervalo de tempo em que os animais permaneceram sem claudicar ;TE (tempo do efeito sensitivo): intervalo de tempo, em minutos, que os animais retornam ao grau 3 .

*diferença estatisticamente significativa entre os anestésicos (Bupivacaína 0,5\% e Ropivacaína $0,5 \%$ ) pelo teste de Wilcoxon $(p<0,05)$

Figura 2- Medianas de tempo para resposta anestésica, em minutos, obtida com bupivacaína $0,5 \%$ e ropivacaína $0,5 \%$ sem vasoconstritor para TL (tempo de latência sensitiva) e TLM (tempo de latência para efeito máximo) (A); e para TEM (tempo de duração do efeito máximo) e TE (tempo do efeito sensitivo) (B), após bloqueio dos nervos palmares em equinos com claudicação induzida. 
Sendo: TL (tempo de latência sensitiva):

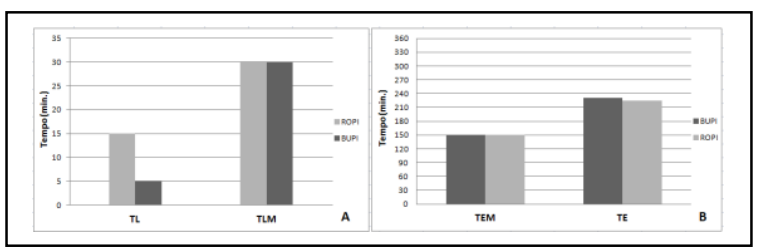

Avaliando os tempos obtidos em relação aos encontrados na literatura, nota-se que TL da bupivacaína no nervo palmar de equinos foi de 5 minutos, bem menores que os 10 a 30 minutos citados para equinos por Lamount (2008) e aos 57 minutos em plexos braquiais de cães citados por Wakoff et al. (2013). Esse fato pode ser justificado pela infiltração mais próxima do nervo devido à neurolocalização, diminuindo o tempo de difusão no espaço subcutâneo para ambos os fármacos, no entanto a lipossolubilidade menor da ropivacaína não promoveu TL menor, sendo necessários mais estudos para maiores esclarecimentos. Ainda, o tempo de latência sensitiva apresentado no estudo de Wakoff et al.(2013) é conceitualmente semelhante ao TLM, que também foi inferior nesse experimento, com tempo de $30(\mathrm{Dl}=15)$ minutos. Já em relação ao TE observado de $230(\mathrm{Dl}=0)$ minutos, está dentro do tempo proposto por Lamount (2008) e inferior aos 768,5 minutos obtidos por Wakoff et al. (2013) em plexos braquiais de cães.

Figura 3- Avaliação da resposta anestésica, em minutos, após bloqueio dos nervos palmares em equinos com claudicação induzida, obtida com bupvacaína $0,5 \%$ (A) e ropivacaína $0,5 \%$ (B) sem vasoconstritor

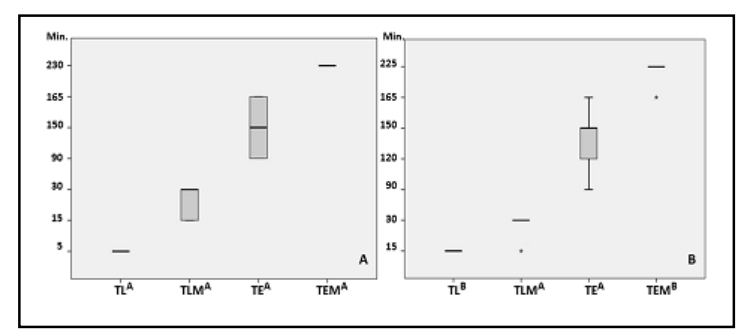

intervalo de tempo, em minutos, entre a infiltração do fármaco e a diminuição do grau de claudicação (grau 2 ou 1); TLM (tempo de latência para efeito máximo): intervalo de tempo, em minutos, entre a infiltração do fármaco até a ausência de claudicação (grau 0); TE (tempo do efeito sensitivo): intervalo de tempo, em minutos, que os animais retornam ao grau 3; TEM (tempo de duração do efeito máximo): intervalo de tempo em que os animais permaneceram sem claudicar. " outiliers: equinos 1 (TLM) e 4 (TEM)

Letras maiúsculas diferentes (em cada parâmetro) entre os diferentes tratamentos diferem $(p<0,05)$ entre si pelo teste de Wilcoxon

C Já avaliando a ropivacaína, o TL de $15(\mathrm{Dl}=0)$ minutos e o TE de 225 ( $\mathrm{DI}=30)$ minutos estão dentro dos citados por Lamount (2008) em equinos, porém ainda bem menores aos 75,38 minutos de latência e 296,9 minutos de duração de bloqueio sensitivo em plexos braquiais de cães (Wakoff et al., 2013).

A cardiotoxicidade da bupivacaína, segundo LUNA (1998) e NOLAN (2000), só é observada em doses acima de $2 \mathrm{mg} / \mathrm{kg}$, sendo que neste experimento a dose total utilizada foi de $50 \mathrm{mg}$ por animal, variando de 0,135 a $0,217 \mathrm{mg} / \mathrm{Kg}$.

\section{CONCLUSÃO}

A utilização de $5 \mathrm{~mL}$ bupivacaína $0,5 \%$ sem vasoconstrictor em nervos palmares de equinos conferiu menor tempo de latência e maior tempo de analgesia em relação ao mesmo volume de ropivacaína $0,5 \%$, sendo mais indicada para a técnica de anestesia perineural em equinos hígidos. 


\section{AGRADECIMENTOS}

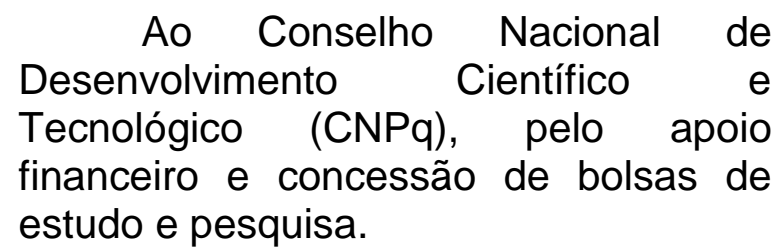

\section{COMITÊ DE ÉTICA E BIOSSEGURANÇA}

A pesquisa foi aprovada pelo Comitê de Ética em Pesquisa da Universidade Federal de Alagoas, conforme protocolo $\mathrm{n}^{\circ}$ : 010480/2011-29.

\section{REFERÊNCIAS}

ADAMI, C.; BERGADANO,A.; BRUCKMAIER,R.M. et al. Sciatic- femoral nerve block with bupivacaine in goats undergoing elective stifle arthrotomy. The Veterinary Journal, v.188, p. 53-57, 2011.

BEATO, L.; CAMOCARDI, G.; IMBELLONI,L.E. et al. Bloqueio do plexo braquial pela via posterior com uso de neuroestimulador e ropivacaína a $0,5 \%$. Rev. Bras.Anestesiol, Rio de Janeiro, v. 55 , n. 4, p. 421-428, 2005.

DRIESSEN, B.; SCANDELLA,M.; ZARUCCO,L. Development of a techinique for continuous perineural blockade of palmar nerves in the distal equine thoracic limb. Veterinary Anesthesia and Analgesia, v.35, p.432-448, 2008.

ESCODRO, P.B. Avaliação da eficácia e segurança clínica de uma formulação neurolítica injetável para uso perineural em eqüinos. 2011. 147f. Tese (doutorado)- Instituto de Química e Biotecnologia. Universidade Federal de Alagoas.

ESCODRO, P.B.; TONHOLO, J.; THOMASSIAN, $A$. et al. Considerações acerca dos fármacos neurolíticos na medicina equina. Revista Brasileira de Medicina Equina, v.35, p.26-31, 2011.

FELDMAN, H.S. Toxicity of Local Anesthetic Agents. In: RICE, A.S.; FISH, K.J. Anesthetic Toxicity. New York, Raven Press, 1994, p.107133.

FUTEMA, F.; FANTONI, D.T.; AULER JUNIOR, J.O.C. et al.. Nova técnica de bloqueio do plexo braquial em cães. Ciência Rural, Santa Maria, v. 29, n. 1, p. 63-69, 1999.

GILBSON, K.T.; STASHAK, T.S. Using perineural anesthesia to localize equine lameness. Vet. Med., v.84, n.11, p.1082-1086, 1989.
KRUIJT SPANJER, M. R.; BAKKER, N.A.; ABSALOM, A.R. Pharmacology in the elderly and newer anaesthesia drugs. Best Pract Res Clin Anaesthesiol, Amsterdam, v. 25, n. 3, p. 355-365, 2011.

LAMOUNT, L. Anestésicos Locais. In: DOHERTY, T.; VALVERDE, A. Manual de Anestesia e Analgesia em Equinos. São Paulo: Roca, 2008, p.144-154.

LOFSTROM, J.B. The effect of local anesthetics on the peripheral vasculature. Reg Anesth, v.17, p. 1-11, 1992.

LOPES , M.A.F. Detecção de claudicação em equinos com um equipamento portátil à base de sensores inerciais sem fio. Revista Brasileira de Medicina Equina, v.37, p.10-16, 2011.

LUNA, S.P.L. Anestesias perineurais e regionais em eqüinos. Revista de Educação Continuada do CRMV-SP. São Paulo, fasc.1, v.1, p.24-30, 1998.

MOYER, W.; SHUMACHER,J.; SHUMACHER, J. A Guide to Equine: Joint Injection and Regional Anesthesia. Pennsylvania: Veterinary Learning Systems, 2007, 110p.

NICOLETTI, J. L. M.; ESCODRO, P.B.; HUSSNI, C.A. et. al. Estudo comparativo experimental da injeção perineural do álcool benzílico $0,75 \%$ e do álcool etílico absoluto nos nervos palmares de equinos. Braz. J. Vet. Res. Anim. Sci., São Paulo, v. 44 , n. 6 , p. $401-407,2007$.

NOLAN, A.M. Pharmacology of analgesic drugs. In: FLECKNELL, P.; WATERMAN-PEARSON, A.. Pain management in animals. London: W.B. Saunders, 2000. p.21 -52.

STASHAK, T. S. Adam's lameness in horse. 4.ed. São Paulo: Roca, 2002. 906p.

WAKOFF, T.I.; MANCALHA R.; SOUZA, M.S. et al. Bupivacaína $0,25 \%$ versus ropivacaína $0,25 \%$ no bloqueio do plexo braquial em cães da raça Beagle. Semina: Ciências Agrárias, v.34, n.3, p. 1259-1272, 2013.

ZARUCCO, L.; DRIESSEN, B.; SCANDELA, M. et al. Sensory nerve conduction and nociception in the equine lower forelimb during perineural bupivacaina infusion along the palmar nerves. The Canadian Journal of Veterinary Research, v.74, p.305-313, 2010. 